\title{
Disseminated Blastomycosis during Pregnancy at 11 Weeks Gestation
}

\author{
Caroline E. Dillon1, Paula A. McKenzie2 \\ ${ }^{1}$ Department of Pharmacy, Geisinger Medical Center, Danville, PA, USA \\ ${ }^{2}$ Department of Infectious Diseases, Geisinger Medical Center, Danville, PA, USA \\ Email: *pamckenzie1@geisinger.edu
}

How to cite this paper: Dillon, C.E. and McKenzie, P.A. (2022) Disseminated Blastomycosis during Pregnancy at 11 Weeks Gestation. Open Journal of Obstetrics and Gynecology, 12, 118-123.

https://doi.org/10.4236/ojog.2022.122012

Received: November 22, 2021

Accepted: February 7, 2022

Published: February 10, 2022

Copyright $\odot 2022$ by author(s) and Scientific Research Publishing Inc. This work is licensed under the Creative Commons Attribution International License (CC BY 4.0).

http://creativecommons.org/licenses/by/4.0/

\begin{abstract}
Blastomyces dermatitidis is a dimorphic fungus that typically causes disease in immunocompromised hosts, but can affect immunocompetent patients as well. Here we present a case of disseminated blastomycosis in a pregnant patient at 11 weeks gestation who was successfully treated with systemic antifungal therapy. Data regarding treatment of blastomycosis in pregnancy is limited, and choice of antifungal agent remains challenging due to uncertainties regarding fetal toxicity.
\end{abstract}

\section{Keywords}

Blastomyces, Itraconazole, Amphotericin, Antifungal

\section{Introduction/Background}

Blastomycosis is typically caused by direct cutaneous contact or inhalation of the dimorphic fungi Blastomyces dermatitidis. Incidence of these infections is typically most common in midwestern, south central, and southeastern regions of the United States, as well as areas of Canada that border the St. Lawrence River Valley and the Great Lakes [1] [2]. Manifestations of blastomycosis are variable and can include multiple organ systems. Most commonly affected are lungs and skin, but infection can also disseminate to CNS, bone, genitourinary, liver, and spleen [1]. B. dermatitidis can cause infection in both immunocompetent and immunocompromised patients. Though disease can be caused in immunocompetent hosts, case series have found those with an impaired immune system are more likely to have disseminated severe disease [3] [4]. Since pregnancy partially modulates the maternal immune system, there is potential for more severe disease if contracted [5]. There is limited data regarding treatment of disseminated 
blastomycosis in pregnancy, especially early in gestational age. Antifungal agents remain a challenge in pregnancy due to uncertainties regarding fetal toxicity and altered maternal pharmacokinetics that may affect efficacy or increase maternal and fetal toxicity. Here we present a case of disseminated blastomycosis in a patient at 11 weeks gestation.

\section{Case}

A 24-year-old female (full demographics included in Table 1), G3P1 at 11 weeks pregnant initially presented with dizziness, shortness of breath, chest pain which radiated to right arm and worsened when laying on right side and vomiting for 5 days. Additionally, reported unintentional weight loss for unspecified amount of time. Upon presentation, a respiratory viral panel (RVP) including SARS-CoV-2 was sent and negative. A CT-PE study was also done and was negative for acute pulmonary embolus but found a mass like consolidation in the anterior left upper lobe with focal area of necrosis concerning for necrotizing pneumonia (Figure 1).

Table 1. Patient demographics.

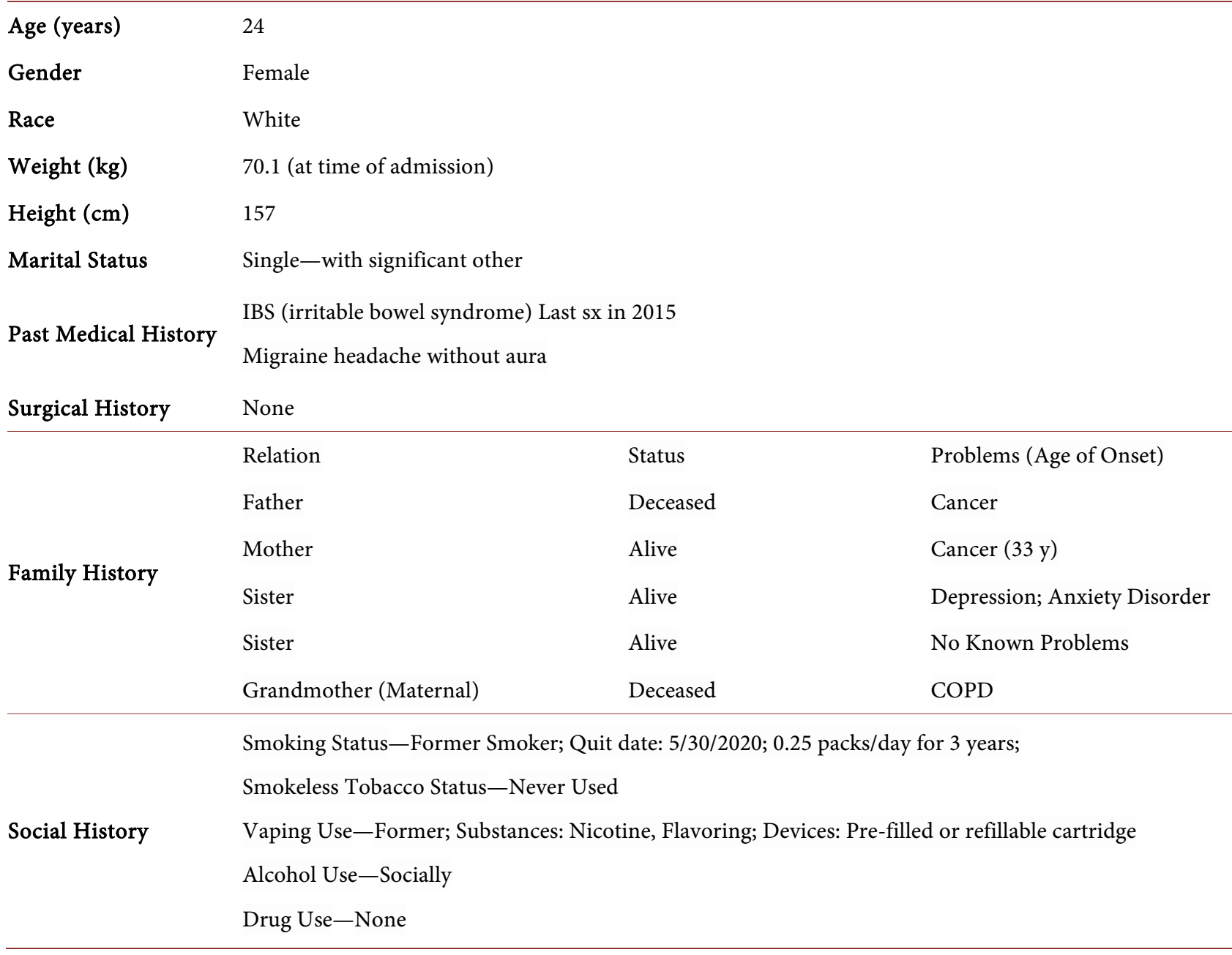




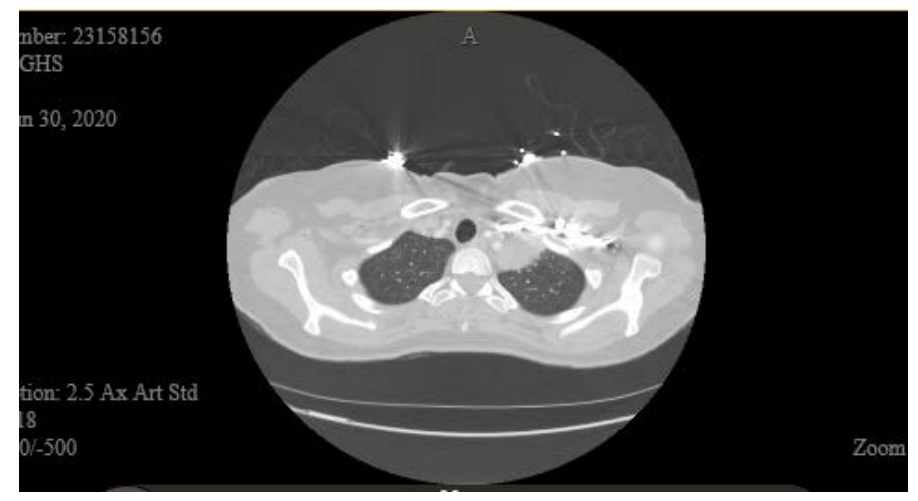

Figure 1. CT image of lung.

At that time, blood and respiratory cultures were collected and were negative. The patient was discharged on piperacillin-tazobactam and vancomycin for 4 - 6 weeks with a plan to repeat the CT chest in 6 weeks and evaluation by thoracic medicine for possible bronchoscopy. Approximately 1 week after the patients discharge, her serum creatinine increased from baseline $0.4-0.5 \mathrm{mg} / \mathrm{dL}$ to 1.5 $\mathrm{mg} / \mathrm{dL}$ and she was instructed to hold piperacillin-tazobactam and vancomycin. Antibiotics were held for about a week while patients acute kidney injury (AKI) resolved and then patient resumed piperacillin-tazobactam monotherapy. One day after resuming, our patient was re-admitted with complaints of hemoptysis, poor appetite and a lesion on her right hip. The patient underwent bronchoscopy with biopsy of the lung mass (Figure 2) which showed benign lung parenchyma with active chronic inflammation and multinucleated giant cells, suggestive of non-necrotizing granuloma; fungal organisms (budding yeast form) were also observed. Special stains for fungus and acid-fast bacteria were performed and rare broad-based budding yeast forms were identified, measuring $12-15$ $\mu \mathrm{m}$, consistent with fungal organisms, favoring Blastomyces species.

Additionally, a punch biopsy of the right hip lesion (Figure 3) was obtained and returned with yeast-like organisms within multinucleated giant cells consistent with blastomycosis. Fungal tissue culture from the lesion was also sent and resulted as $B$. dermatitidis.

Blastomycosis has been reported infrequently in pregnancy. Per the 2008 IDSA guideline recommendations for treatment of blastomycosis, azole antifungal agents should be avoided, and amphotericin B remains the systemic agent of choice despite its well-established toxicity [2]. Data has demonstrated similar rates of nephrotoxicity in pregnant and non-pregnant patients [6]. Liposomal and lipid complex formulations of amphotericin B have also been shown to be safe and effective in pregnancy and are typically recommended over conventional forms due to side effect profile. Amphotericin B does cross the placenta, but no teratogenic effects have been seen in animal studies [7] [8]. Though there is limited human data on amphotericin B lipid complex and amphotericin B liposomal formulations in pregnancy, they still remain the systemic agents of choice for treatment of blastomycosis and should be used with careful risk-benefit assessment. 


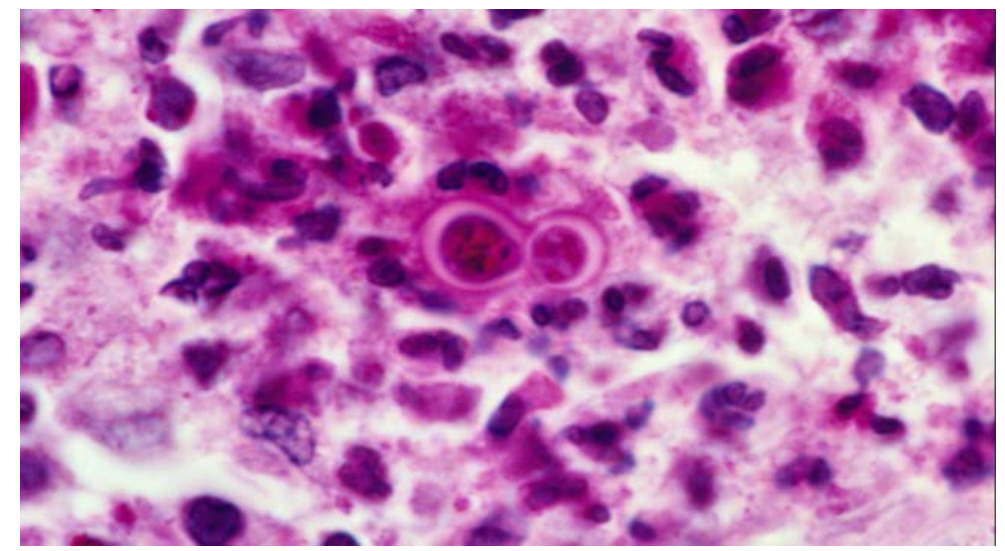

Figure 2. Pathology image from biopsy of lung mass.

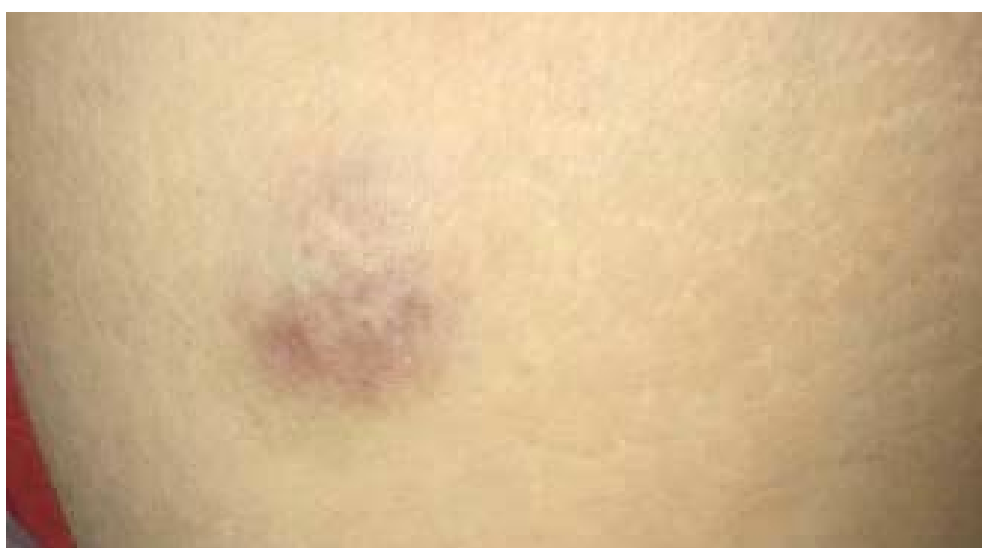

Figure 3. Image of hip lesion.

Itraconazole is an additional standard of care drug for the treatment of blastomycosis in non-pregnant patients. Conversely to amphotericin B lipid complex which is recommended in pregnancy, guidelines recommend against the use of itraconazole due to risk of teratogenicity [2]. Itraconazole was found to cause a dose-related increase in maternal toxicity, embryotoxicity, and teratogenicity in animal studies [9] and should be avoided in pregnancy especially during the first trimester [2]. A 2009 study in Italy compared a control group of non-pregnant women to a group that was exposed to itraconazole in the first trimester and found no significant difference in congenital abnormalities, however did discover a higher rate of induced and spontaneous abortions [7] [10]. A recent meta-analysis in 2020 looked at fetal outcomes after maternal exposure to oral fluconazole and itraconazole. The analysis found that oral itraconazole or fluconazole were not associated with an increased risk of overall birth defects, spontaneous abortion, or stillbirth. However, the agents may be associated with a possible increased risk of specific birth defects (eye defect, limb defect, nervous system, digestive system, urinary system [11]. Despite the increase in safety data, overall the use of itraconazole is still not recommended in pregnancy, especially in the first trimester. Manufacturer labeling also recommends contraception during treatment with itraconazole and for at least 2 months after [9]. 
Upon biopsy and tissue culture confirmation of $B$. dermatitidis, piperacillintazobactam was discontinued and a multi-disciplinary decision (including the patient and father of the baby) was made to start the patient on amphotericin $B$ lipid complex (Abelcet) infusion $300 \mathrm{mg}(5 \mathrm{mg} / \mathrm{kg})$ once daily for 2 weeks followed by itraconazole $200 \mathrm{mg}$ twice daily for a total of 12 months of treatment. Approximately one hour into the first amphotericin infusion patient experienced nausea, vomiting, chills and rigors. First infusion was stopped, and patient was given IV diphenhydramine and had resolution of symptoms. The following day, infusion time was adjusted to run at a slower rate, and pre-medication with IV diphenhydramine, IV prochlorperazine, and acetaminophen was ordered with next infusion. Unfortunately, patient still experienced similar symptoms and expressed her desire to stop amphotericin infusions. Additionally, the patient was recovering from an acute kidney injury secondary to earlier vancomycin and piperacillin/tazobactam use as previously described in this case. The patient was once again notified of itraconazole risks to fetus, and multi-disciplinary decision was made to move forward and complete a 12 month treatment course with itraconazole monotherapy. She tolerated this regimen without difficulty and delivered a healthy (7l bs $8.6 \mathrm{oz}$ ) baby boy via spontaneous vaginal delivery. The patient was evaluated at her follow-up appointment with infectious disease at which point the 12 month itraconazole regimen was scheduled to be completed.

\section{Conclusions}

While the treatment of disseminated blastomycosis in pregnancy has not been widely studied, the general consensus is that amphotericin is the drug of choice [12]. This approach however is not always feasible as in the case of our patient, who was diagnosed in her first trimester and would have had to be on amphotericin for another 6 months if she had been able to tolerate the drug.

She unfortunately did not tolerate amphotericin leaving the medical team with a dilemma as to what to treat her with, bearing in mind that the azoles have embryotoxic and teratogenic properties making them contra-indicated in pregnancy. We had a good outcome with itraconazole and perhaps more research needs to be done into appropriate alternative treatment for pregnant patients with disseminated blastomycosis.

\section{Conflicts of Interest}

The authors declare no conflicts of interest regarding the publication of this paper.

\section{References}

[1] Saccente, M. and Woods, G.L. (2010) Clinical and Laboratory Update on Blastomycosis. Clinical Microbiology Reviews, 23, 367-381. https://doi.org/10.1128/CMR.00056-09

[2] Chapman, S.W., Dismukes, W.E., Proia, L.A., Bradsher, R.W., Pappas, P.G., Threlkeld, M.G. and Kauffman, C.A. (2008) Clinical Practice Guidelines for the Man- 
agement of Blastomycosis: 2008 Update by the Infectious Diseases Society of America. Clinical Infectious Diseases, 46, 1801-1812. https://doi.org/10.1086/588300

[3] Pappas, P.G., Threlkeld, M.G., Bedsole, G.D., Cleveland, K.O., Gelfand, M.S. and Dismukes, W.E. (1993) Blastomycosis in Immunocompromised Patients. Medicine, 72, 311-325. https://doi.org/10.1097/00005792-199309000-00003

[4] McBride, J., Sterkel, A., Matkovic, E. and Gauthier, G. (2017) Clinical Manifestations and Outcomes in Immunocompetent and Immunocompromised Patients with Blastomycosis. Open Forum Infectious Diseases, 4, S718. https://doi.org/10.1093/ofid/ofx163.1933

[5] Mor, G. and Cardenas, I. (2010) The Immune System in Pregnancy: A Unique Complexity. American Journal of Reproductive Immunology, 63, 425-433. https://doi.org/10.1111/j.1600-0897.2010.00836.x

[6] Moudgal, V.V. and Sobel, J.D. (2003) Antifungal Drugs in Pregnancy: A Review. Expert Opinion on Drug Safety, 2, 475-483. https://doi.org/10.1517/14740338.2.5.475

[7] Pilmis, B., Jullien, V., Sobel, J., Lecuit, M., Lortholary, O. and Charlier, C. (2015) Antifungal Drugs during Pregnancy: An Updated Review. Journal of Antimicrobial Chemotherapy, 70, 14-22. https://doi.org/10.1093/jac/dku355

[8] Abelcet (Amphotericin Lipid Complex) [Package Insert]. Indianapolis, IN. Exelead, Inc: Nov. 2018.

[9] Sporanox (Itraconazole) [Package Insert]. Titusville, NJ: Janssen Pharmaceuticals Inc. May 2018.

[10] De Santis, M., Di Gianantonio, E., Cesari, E., Ambrosini, G., Straface, G. and Clementi, M. (2009) First-Trimester Itraconazole Exposure and Pregnancy Outcome: A Prospective Cohort Study of Women Contacting Teratology Information Services in Italy. Drug Safety, 32, 239-244. https://doi.org/10.2165/00002018-200932030-00006

[11] Liu, D., Zhang, C., Wu, L., Zhang, L. and Zhang, L. (2020) Fetal Outcomes after Maternal Exposure to Oral Antifungal Agents during Pregnancy: A Systematic Review and Meta-Analysis. International Journal of Gynecology \& Obstetrics, 148 , 6-13. https://doi.org/10.1002/ijgo.12993

[12] Baker, T., Patel, A., Halteh, P., Toussi, S.S., DeLaMora, P., Lipner, S., Schuetz, A.N. and Hartman, B. (2017) Blastomycosis during Pregnancy: A Case Report and Review of the Literature. Diagnostic Microbiology and Infectious Disease, 88, 145-151. https://doi.org/10.1016/j.diagmicrobio.2017.02.015 